\title{
Analysis of adiabatic heating in high strain rate torsion tests by an iterative method: application to an ultrahigh carbon steel
}

\author{
J. Castellanos ${ }^{1}$, I. Rieiro ${ }^{1}$, M. Carsí ${ }^{2}$, J. Muñoz ${ }^{1} \&$ O. A. Ruano ${ }^{2}$ \\ ${ }^{1}$ Department of Mathematics, University of Castilla - La Mancha, Spain \\ ${ }^{2}$ Department of Physical Metallurgy, \\ National Center for Metallurgical Research (C.E.N.I.M.), Spain
}

\begin{abstract}
An iterative algorithm has been developed to establish the adiabatic heating correction of flow curves for torsion tests of an ultrahigh carbon steel containing $1.3 \% \mathrm{C}$. High temperatures (1223 to $1473 \mathrm{~K})$ and high strain rates $(2,5,10$ and $26 \mathrm{~s}^{-1}$ ) were used. The curves are corrected in a finite and discrete set of strain data by means of parametric derivatives and integration on the initial curve without correction. The process is repeated until the termination tolerance for the stress is less than $10^{-2} \mathrm{MPa}$. Usually, four iterations are needed to reach this tolerance. The corrections are bounded by the maximum of mechanical energy available to be converted into heat. The corrections are carried out until a true strain $\varepsilon=4$ in order to avoid the effects of flow localization in the material.

Keywords: adiabatic heating, torsion test, modelling, simulation, Garofalo equation, hot working.
\end{abstract}

\section{Introduction}

Torsion tests at high temperatures and strain rates of materials usually show a strong increment of temperature during the test above the programmed temperature that is attributed to adiabatic heating [1]. The temperature correction due to adiabatic heating has been discussed in various works [1-6]. The following expression is usually considered:

$$
\Delta T_{i}(\varepsilon)=\frac{\eta \cdot A}{\rho \cdot C} \cdot \int_{\varepsilon_{\max }}^{\varepsilon} \sigma(\varepsilon) \cdot d \varepsilon
$$


where $\mathrm{T}$ is the test temperature, $\mathrm{C}$ is the specific heat capacity, $\rho$ is the density, $\eta$ and $\mathrm{A}$ are efficiency coefficients of the energetic conversions and $\sigma(\varepsilon)$ is the stress-strain relation.

Some authors assume a variable energy performance in eqn (1) [2] or a constant one [6]. Other authors use the relation $\sigma(\varepsilon)$ without considering the intrinsic error due to the adiabatic heating itself [3]. In general, it is not considered that determination of the term $\sigma(\varepsilon)$ implies derivatives at constant temperature, which is not true under the effect of adiabatic heating. These derivatives appear in the calculation of the strain rate sensitivity and the strain hardening coefficients. In addition, constant values for $\rho$ and $\mathrm{C}$ are used in the entire working range.

In this work, we consider the following expression for determining the true value of the corrected relation $\sigma^{c}$, in contrast to the experimental value $\sigma^{w c}$ :

$$
\sigma_{i}^{c}(\varepsilon)=\sigma_{i}^{w c}(\varepsilon)-\left.\frac{\partial \sigma_{i}^{w c}}{\partial T}(\varepsilon, \dot{\varepsilon}, T)\right|_{\varepsilon, \dot{\varepsilon}} \cdot \Delta T_{i}(\varepsilon)+\Delta \xi
$$

where $\Delta \xi$ is the error associated to the experimental value of the stress that should be bounded to avoid wrong answers.

The goal of this work is to design a modular and iterative algorithmic method that guarantees the convergence of the experimental function $\sigma_{i}^{w c}(\varepsilon)$ to the nominal function $\sigma_{i}^{c}(\varepsilon)$. This method is based in eqns (1) and (2). The validity ranges of the algorithms are adjusted taken into account physical fundaments on flow localization $[3,5]$ and bounds of the performance for the conversion on mechanical energy into heat.

\section{Material and experimental procedure}

The UHC-1.3\%C steel studied in this investigation has the following composition: $1.3 \% \mathrm{C}, 0.5 \% \mathrm{Mn}, 0.6 \% \mathrm{Si}, 0.18 \% \mathrm{Cr}$ and balance $\mathrm{Fe}$ [1]. The manganese was added to neutralize the deleterious effects of sulphur and phosphorus. The steel was obtained at Sidenor Industry as a cast of 8 litres by means of an induction furnace. The as-cast ingot was initially soaked at $1050^{\circ} \mathrm{C}$ and forged into a bar of $60 \mathrm{~mm} \times 55 \mathrm{~mm}$ cross section.

Simulation of the forming process of forged parts was carried out by means of torsion tests. An induction furnace heats the test sample and the temperature is continuously measured by means of a two-color pyrometer. A silica tube with argon atmosphere ensures protection against oxidation. A helium atmosphere is used to obtain, after testing, a cooling rate of $325 \mathrm{~K} / \mathrm{s}$.

The torsion samples have an effective gage length of $17 \mathrm{~mm}$ and a radius of 3 $\mathrm{mm}$. The density and specific heat are $7800 \mathrm{~kg} \cdot \mathrm{m}^{-3}$ and $670 \mathrm{~J} \cdot \mathrm{kg}^{-1} \cdot \mathrm{K}^{-1}$, respectively. Strain rates in the range 2 to $26 \mathrm{~s}^{-1}$ were used. The temperature 
range was 900 to $1200{ }^{\circ} \mathrm{C}$. The samples were deformed in a SETARAM torsion machine at CENIM (National Center for Metallurgical Research) in Madrid, Spain.

\section{Theoretical approach}

Two main processes limit the conversion of mechanical energy into heat in an adiabatic framework: changes in the internal energy of the material and flow localization. Both processes are related to the start of catastrophic failure [2-4]. A differential expression for the first law of thermodynamics $\sigma \cdot d \varepsilon=\rho \cdot c \cdot d T+d u_{m s t}$ where $d u_{m s t}$ is the variation of the microstructural internal energy can be considered. The plastic work carried out by the material is transformed into heat that is used to increase the internal energy of the material. Some authors assume $d u_{m s t}=0$ [3]. Other authors consider $d u_{m s t} \neq 0$ leading to the general expression [2]:

$$
d T=\frac{\sigma \cdot d \varepsilon}{\rho \cdot c} \cdot\left(1-\frac{1}{\sigma} \cdot \frac{d u_{m s t}}{d \varepsilon}\right)=\frac{\sigma \cdot d \varepsilon}{\rho \cdot c} \cdot \eta(\varepsilon, \dot{\varepsilon}, T)
$$

where $\eta(\varepsilon, \dot{\varepsilon}, T)=\left(1-(1 / \sigma) \cdot\left[d u_{m s t} / d \varepsilon\right]\right)$ is the performance of the conversion and it is variable. A constant value for $\eta$ of 0,90 or 0,95 may be taken but an iterative procedure would be necessary to eliminate the effect of this approximation.

The approach of Prasad et al. [4] is convenient to estimate the upper limit bound of the increase of temperatures due to adiabatic heating. A simple constitutive equation for the energy dissipation is the following:

$$
\sigma \cdot \dot{\varepsilon}=\int_{0}^{\dot{\varepsilon}} \sigma \cdot d \dot{\varepsilon}+\int_{0}^{\sigma} \dot{\varepsilon} \cdot d \sigma=G+J
$$

where $\mathrm{G}$ is the dissipator content and $\mathrm{J}$ is the dissipator co-content. Part of the power dissipated by the plastic flow, G, can be converted into heat. The quantity $\mathrm{J}$ is related to the processes of form change. The limit for $\mathrm{G}$ is $G_{\max }=\left(\sigma_{\max } \cdot \dot{\varepsilon}_{\max }\right) / 2$. The following expression can be deduced from eqn (4):

$$
\Delta T_{\max }=\frac{\eta \cdot G_{\max }}{\rho \cdot C \cdot V}
$$

that represents the limit for the adiabatic $\Delta T$ in a volume $\mathrm{V}$.

The increment in temperature can be expressed as [3]:

$$
\Delta T=\frac{\eta}{\rho \cdot c} \int_{\varepsilon_{0}}^{\varepsilon_{p}^{\prime}} \sigma \cdot d \varepsilon
$$


where $\eta$ is constant and $\varepsilon_{p}{ }^{\prime}$ is the deformation limit where the plastic instability starts [3]. Therefore, a critical deformation can be considered above which it is not possible to apply this kind of corrections [5]. Values of $d T / d \varepsilon=165 \mathrm{~K}$ from eqn (6) are obtained for the UHC-1.3\%C steel.

Assuming the analysis of Armstrong et al. [3] and considering the stressstrain relation $\sigma=K_{1} \cdot \varepsilon^{\theta}$, together with the definition of the stress exponent, n, in the Garofalo equation and the definition of constant strain rate tests, $\varepsilon_{p}=\dot{\varepsilon}_{p} \cdot t$, the condition for plastic instability gives the following equation:

$$
\left[\frac{d T}{d \varepsilon_{p}}\right]_{\varepsilon_{p}^{\prime}}=\left(\theta+\frac{1}{n}\right) /\left(\varepsilon_{p}^{\prime} \cdot \beta\right)
$$

Under stability conditions, $d \sigma>0$. The instability starts at $d \sigma=0$, that can be expressed as $[7,8]$ :

$$
d \sigma=\left.\left(\frac{\partial \sigma}{\partial \varepsilon}\right)\right|_{\varepsilon, \dot{\varepsilon}} \cdot d \varepsilon+\left.\left(\frac{\partial \sigma}{\partial \dot{\varepsilon}}\right)\right|_{\varepsilon, \dot{\varepsilon}} \cdot d \dot{\varepsilon}+\left.\left(\frac{\partial \sigma}{\partial T}\right)\right|_{\varepsilon, \dot{\varepsilon}} \cdot d T=0
$$

Under adiabatic heating conditions, and assuming $\dot{\varepsilon}$ constant, by means of the Garofalo equation $\left(\dot{\varepsilon}=A \cdot e^{-\frac{Q}{R \cdot T}} \cdot(\sinh (\alpha \cdot \sigma))^{n}\right)$ it is obtained [7] that $\left.(\partial \sigma / \partial \varepsilon)\right|_{\dot{\varepsilon}, T}=-\theta \cdot \sigma / \varepsilon$ and $\left.(\partial \sigma / \partial T)\right|_{\dot{\varepsilon}, \varepsilon}=\sigma \cdot\left(-Q / R \cdot T^{2}\right)$. Taken these expressions into the plastic instability condition, an expression for the flow stress at which the instability starts, $\sigma_{i n}$, can be obtained:

$$
\sigma_{i n}=-\frac{\theta \cdot R \cdot T^{2} \cdot \rho \cdot c}{\eta \cdot Q \cdot \varepsilon}
$$

This expression will be applied later to the UHC-1.3\%C steel.

\section{Basic methodology}

The following assumptions are used in the algorithm developed in this work: 1) adiabatic conditions in the deformation process, 2) $\rho$ and $\mathrm{C}$ do not vary with $\mathrm{T}$, 3) $\eta$ and $\mathrm{A}$ are constant with strain, and 4) adiabatic heating has an important effect from the peak stress of the curve $\sigma(\varepsilon)$, to a value $\varepsilon_{f}$.

For a given test at constant $\dot{\varepsilon}_{j}$ and $T_{s}$, the initial temperature, and for a given value of $\varepsilon$, the uncorrected stress, $\sigma_{0}{ }^{w c}$ (experimental stress), can be expressed as a function of the corrected stress, $\sigma_{0}{ }^{c}$, as: 


$$
\sigma_{0}{ }^{w c}(T)=\sigma_{0}^{c}(T+\Delta T)
$$

Applying Taylor expansion of the function $\sigma_{0}^{c}$ in eqn (10) about the point T:

$$
\sigma_{0}^{c}(T)=\sigma_{0}{ }^{w c}(T)-\frac{\partial \sigma_{0}{ }^{w c}(T)}{\partial T} \Delta T
$$

where it is assumed that $\partial \sigma_{0}^{w c} / \partial T \approx \partial \sigma_{0}^{c} / \partial T$.

On the other hand, the temperature increment for each measured strain $\varepsilon_{i}$, for a given test at constant $\dot{\varepsilon}_{j}$, can be expressed as:

$$
\Delta T_{0}\left(\varepsilon_{i}\right)=\frac{\eta \cdot A}{\rho \cdot C} \cdot \int_{\varepsilon_{0}=\varepsilon_{\max }}^{\varepsilon_{i}} \sigma_{0}^{w c}\left(\varepsilon, \dot{\varepsilon}_{j}, T\right) \cdot d \varepsilon
$$

Substituting eqn (12) in eqn (11):

$$
\sigma_{0}^{c}\left(\varepsilon_{i}, \dot{\varepsilon}_{j}, T\right)=\sigma_{0}^{w c}\left(\varepsilon_{i}, \dot{\varepsilon}_{j}, T\right)-\frac{\partial \sigma_{0}^{w c}\left(\varepsilon_{i}, \dot{\varepsilon}_{j}, T\right)}{\partial T} \cdot \frac{\eta A}{\rho c} \cdot \int_{\varepsilon_{0}=\varepsilon_{\max }}^{\varepsilon_{i}} \sigma_{0}^{w c}\left(\varepsilon_{i}, \dot{\varepsilon}_{j}, T\right) \cdot d \varepsilon
$$

A single time application of these equations would result in a value of $\sigma_{0}^{c}(\varepsilon, \dot{\varepsilon}, T)$ that is not accurate. This is due, as mentioned in the introduction, to the associated error in the determination of $\sigma_{0}{ }^{w c}$ and consequently in the integral part of eqn (13). Furthermore, calculation of $\partial \sigma_{0}^{w c}(\varepsilon, \dot{\varepsilon}, T) / \partial T$ in eqn (13) is also not accurate since the function $\sigma_{0}{ }^{w c}(\varepsilon)$ is a warped curve in the space $\{\varepsilon, \sigma, T\}$.

To minimize the inaccuracies of the calculation carried out in eqn (13), an iterative algorithm based in eqns (11) and (12) has been developed using as initial value the stress $\sigma_{0}^{c}(\varepsilon, \dot{\varepsilon}, T)$. It is a modular algorithm in three steps. In the first step, the value of $\Delta T_{k}\left(\varepsilon_{i}\right)$ in the iteration $\mathrm{k}$ (from $\mathrm{k}=1$ to the number of iterations) is calculated according to the following expression:

$$
\Delta T_{k}\left(\varepsilon_{i}\right)=\frac{\eta A}{\rho C} \int_{\varepsilon_{\max }}^{\varepsilon_{i}}\left(\sigma_{k-1}^{w c}-\sigma_{k-1}^{c}\right) d \varepsilon
$$

where $\sigma_{k-1}^{w c}$, is the uncorrected stress in the iteration $\mathrm{k}-1$, and $\sigma_{k-1}^{c}$ is the corrected stress in $\mathrm{k}-1$. The integration interval is divided in sufficiently enough small parts. In this work, we have worked step by step with all the output data 
given by the machine. Therefore, a trapezoidal rule is used in order to compute the numerical integration in eqn (14).

In the second step, the partial derivative with respect to $\mathrm{T}$ of the uncorrected stress, $\partial \sigma_{k}^{w c}\left(\varepsilon_{i}, \dot{\varepsilon}_{j}, T\right) /\left.\partial T\right|_{\varepsilon, \dot{\varepsilon}}$ in the iteration $\mathrm{k}$, is calculated by means of the expression:

$$
\frac{\partial \sigma_{k}^{w c}\left(T_{s}, \varepsilon_{i}\right)}{\partial T} \cong \frac{\sigma_{k}^{w c}\left(T_{s+1}, \varepsilon_{i+h}\right)-\sigma_{k}^{w c}\left(T_{s}, \varepsilon_{i+h}\right)}{T_{s+1}-T_{s}}
$$

where $s+1$ refers to the test conducted at the same strain rate but at a temperature $\mathrm{T}_{\mathrm{k}+1}$, belonging to the temperature set $\left\{T_{s}\right\}_{s=1, N}$, that is next in the ascendent sequence. This approximation is good enough since the discretization intervals are small. Finally, the value of the corrected stress in the iteration $\mathrm{k}$ is given as:

$$
\sigma_{k}^{c}\left(\varepsilon_{i}, \dot{\varepsilon}_{j}, T\right)=\sigma_{k}^{w c}\left(\varepsilon_{i}, \dot{\varepsilon}_{j}, T\right)-\frac{\partial \sigma_{k}^{w c}\left(\varepsilon_{i}, \dot{\varepsilon}_{j}, T\right)}{\partial T} \cdot \frac{\eta A}{\rho c} \cdot \Delta T_{k}\left(\varepsilon_{i}\right) \cdot d \varepsilon
$$

The criterion adopted for stopping the algorithm, i.e. the termination tolerance, is $\Delta \sigma \leq 10^{-2} \mathrm{MPa}$ for a given control strain. By means of this procedure, the final measured temperature is reached at a given iteration for a value $\varepsilon$ but the correction is used only up to $\varepsilon_{f}$, a value at which the flow localization is not considered important to distort our correction.

\section{Results and conclusions}

\subsection{Analytical basis}

The results obtained in section 3 for the bound limits are applied in our model to establish the adiabatic correction of the UHC-1.3\%C steel. For this steel, at $\dot{\varepsilon}=10 \mathrm{~s}^{-1}$ and $\mathrm{T}=1323 \mathrm{~K}$, the peak stress is $\sigma_{\max }=100 \mathrm{MPa}$ and, according to eqn (5), $\Delta T_{\max }=172 \mathrm{~K}$. For $\dot{\varepsilon}=2 \mathrm{~s}^{-1}$ and $\mathrm{T}=1323 \mathrm{~K}, \Delta T_{\max }=24 \mathrm{~K}$. This gives an idea of the upper bounds of the uncorrected values.

Using the data of Castellanos et al. for this steel [9], the relation $\sigma(T)=K 2 \cdot e^{-0,0037 \cdot T}$ is obtained. The values of the Garofalo equation are: $\mathrm{Q}=274,3 \mathrm{~kJ} / \mathrm{mol}$ and $\mathrm{n}=4,66$. A value $\bar{\theta} \approx-0.2$ is obtained for an integration on all deformation paths. For comparison, it was obtained for $\varepsilon=1,5, \bar{\theta} \approx-0.11$ and $d T\left(\varepsilon_{p}^{\prime}\right) / d \varepsilon_{p}=28 / \varepsilon_{p}{ }^{\prime} K$ and for $\varepsilon$ next to the peak, $\bar{\theta} \approx 0$ and $d T\left(\varepsilon_{p}^{\prime}\right) / d \varepsilon_{p}=58 / \varepsilon_{p}^{\prime} K$. 
Table 1 shows the limit strains, $\varepsilon_{p}^{\prime}$, for the start of plastic instability or flow localization for the UHC-1.3\% steel. The values were calculated by means of eqn (9) using $\bar{\theta}=-0.2$ and $\eta=0,90$. The values $\varepsilon_{p}^{\prime}$ are determined in the point where the experimental curve $\sigma(\varepsilon)$ cross the curve $\sigma_{i n}(\varepsilon)$. The table shows that the plastic instability is delayed at high temperatures and low strain rates.

It is worth noting that the local instability develops progressively with plastic strain. At $\varepsilon=5$ a clear change of behavior of the flow curves is observed characterized by oscillations of the derivates of the stress with respect to the strain. A value of $\varepsilon_{f}=4$ was chosen because up to this value the corrections were meaningful.

Table 1: Limit strains, $\varepsilon_{p}^{\prime}$, for the start of plastic instability for the UHC$1.3 \% \mathrm{C}$ steel as a function of strain rate and temperature.

\begin{tabular}{|c|c|c|c|c|c|}
\hline $\mathrm{T}(\mathrm{K})$ & from eqn (9) & $\dot{\varepsilon}=2 s^{-1}$ & $\dot{\varepsilon}=5 s^{-1}$ & $\dot{\varepsilon}=10 s^{-1}$ & $\dot{\varepsilon}=26 s^{-1}$ \\
\hline 1223 & $\sigma_{\text {in }}=52.7 / \varepsilon$ & 0.45 & --- & 0.3 & 0.3 \\
\hline 1273 & $\sigma_{\text {in }}=57.1 / \varepsilon$ & 0.6 & 0.5 & 0.45 & 0.4 \\
\hline 1323 & $\sigma_{\text {in }}=61.7 / \varepsilon$ & 0.8 & 0.65 & 0.55 & 0.45 \\
\hline 1373 & $\sigma_{\text {in }}=66.4 / \varepsilon$ & 1.05 & 0.8 & 0.7 & 0.5 \\
\hline 1423 & $\sigma_{\text {in }}=71.3 / \varepsilon$ & 1.5 & 1.2 & 0.9 & 0.7 \\
\hline 1473 & $\sigma_{\text {in }}=76.4 / \varepsilon$ & 1.9 & 1.5 & 1.1 & 0.9 \\
\hline
\end{tabular}

\subsection{Correction of flow curves for the UHC-1.3\%C steel}

The flow curves of the UHC-1.3\% $\mathrm{C}$ steel have been modified to consider the adiabatic heating. The curves were conducted at $\dot{\varepsilon}=2,5,10$, and $26 \mathrm{~s}^{-1}$ and $\mathrm{T}$ from 1223 to $1473 \mathrm{~K}$, with a variation of $50 \mathrm{~K}$. A maximum of four iterations were conducted for the attainment of the final measured temperatures for $\varepsilon \in[6,8]$ although the corrections were carried out up to $\varepsilon_{f}=4$.

Figure 1 shows true stress vs. true strain curves at various strain rates and temperatures for the UHC- $1.3 \% \mathrm{C}$ steel.

The solid lines represent the correction for adiabatic heating according to eqn (12). The corrections agree with those carried out by other authors $[10,11]$. However, somewhat different results were obtained when compared with other investigations where unreliable approximations were conducted [12, 13].

Figure 2 shows the evolution of $\Delta T$, according to eqn (11), with strain for $\dot{\varepsilon}=26 s^{-1}$ at various temperatures. All the temperature increments are inside the bounds established for the maximum increments. The convergence of the iterative algorithm was reached at a maximum of four iterations. 

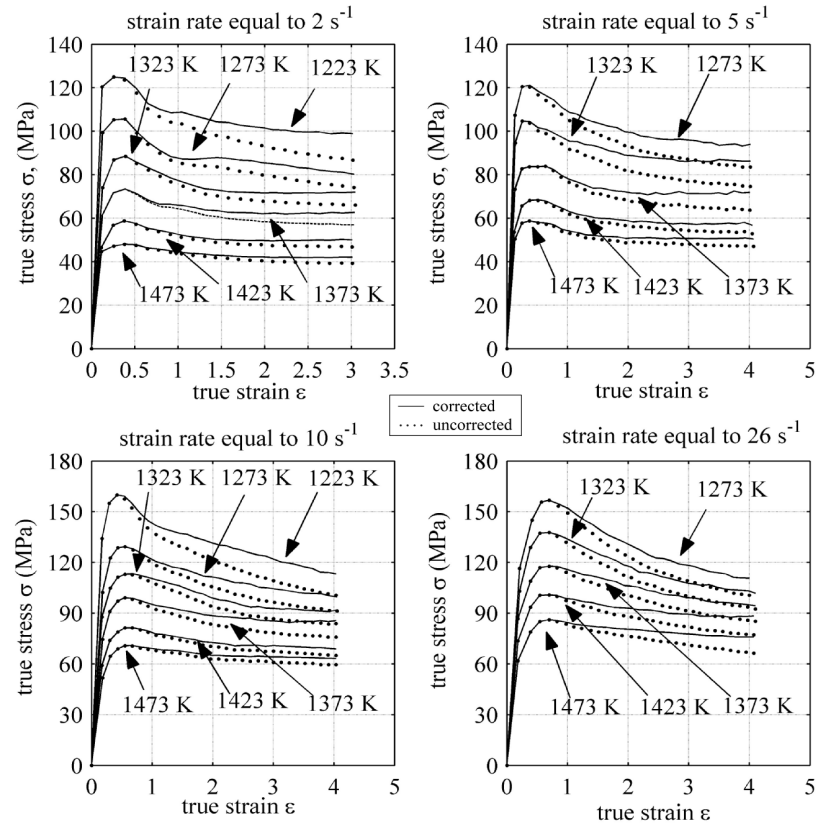

Figure 1: $\quad$ Flow curves for the UHC-1.3\%C steel. Solid lines are corrected curves for adiabatic heating and dotted lines are uncorrected curves.
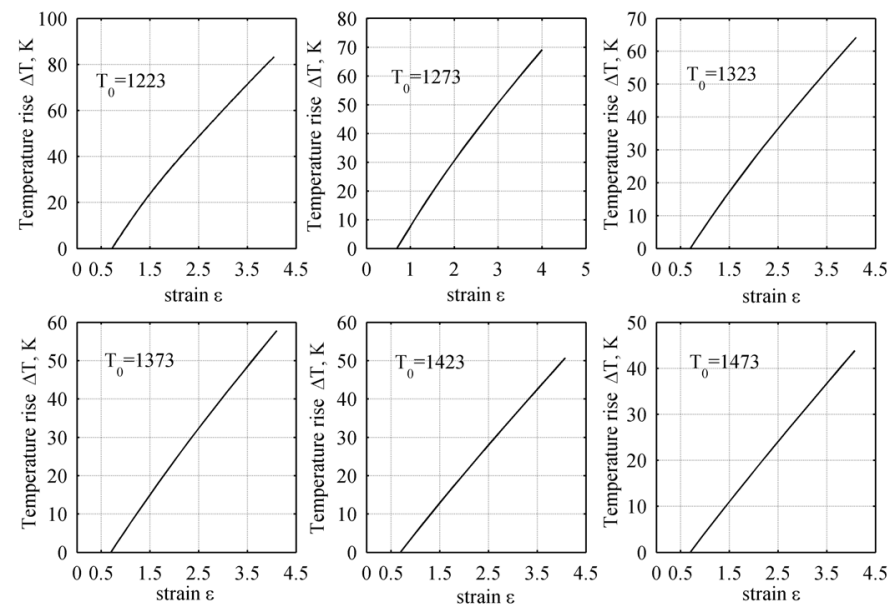

Figure 2: Evolution of $\Delta T$ with strain for several $T_{0}{ }^{\prime} s$ at a $\dot{\varepsilon}=26 \mathrm{~s}^{-1}$. 
Table 2: Values of $\Delta \sigma$ at $\varepsilon=1$ (a) and $\varepsilon=3$ (b) for the corrected tests.

\begin{tabular}{|c|c|c|c|c|c|c|}
\hline (a) & $1223 \mathrm{~K}$ & $1273 \mathrm{~K}$ & $1323 \mathrm{~K}$ & $1373 \mathrm{~K}$ & $1423 \mathrm{~K}$ & $1473 \mathrm{~K}$ \\
\hline$\dot{\varepsilon}=2 s^{-1}$ & 5.38 & 2.06 & 1.91 & 1.85 & 0.9 & 0.9 \\
\hline$\dot{\varepsilon}=5 s^{-1}$ & --- & 3.68 & 2.69 & 1.39 & 1.15 & 1.02 \\
\hline$\dot{\varepsilon}=10 s^{-1}$ & 5.44 & 2.17 & 2.62 & 2.34 & 0.86 & 0.99 \\
\hline$\dot{\varepsilon}=26 s^{-1}$ & 6.54 & 2.76 & 2.49 & 2.37 & 1.41 & 1.28 \\
\hline$(\mathrm{b})$ & $1223 \mathrm{~K}$ & $1273 \mathrm{~K}$ & $1323 \mathrm{~K}$ & $1373 \mathrm{~K}$ & $1423 \mathrm{~K}$ & $1473 \mathrm{~K}$ \\
\hline$\dot{\varepsilon}=2 s^{-1}$ & 12.16 & 6.21 & 5.90 & 5.70 & 3.32 & 2.79 \\
\hline$\dot{\varepsilon}=5 s^{-1}$ & --- & 8.92 & 9.25 & 6.08 & 3.08 & 2.67 \\
\hline$\dot{\varepsilon}=10 s^{-1}$ & 14.11 & 8.46 & 6.32 & 7.57 & 3.48 & 3.29 \\
\hline$\dot{\varepsilon}=26 s^{-1}$ & 16.81 & 9.4 & 7.73 & 7.78 & 7.32 & 6.23 \\
\hline
\end{tabular}

Table 2 shows a summary of all the results obtained in this work. The accumulated values of $\Delta \sigma$ are given for each pair $\{\dot{\varepsilon}, T\}$ at $\varepsilon=1$ and 3. Values at $\varepsilon=5,6$ are higher but were not considered due to flow localization.

It can be concluded that the method, and the implemented algorithm, that we have developed in this work is reliable and convergent. The corrected stressstrain curves are efficient and reliable and take all the experimental data set without the need of average approximations. In addition, the method provides the detailed corrections at the discretization level given by the machine.

The main conclusions of this work are:

1. A new iterative approach for the adiabatic heating correction for torsion tests has been established. It is a natural generalization of a previous approach where the correction was carried out in a single run.

2. The new approach brings an improvement in the precision of the corrected flow curves. The relative errors associated to determination of the experimental stresses are minimized.

3. The temperature increments obtained for the $\mathrm{UHC}-1.3 \% \mathrm{C}$ steel are inside the bounds established for the maximum increments due to adiabatic heating.

\section{Acknowledgement}

The work was carried out through the Project PBC-05-010-1 from JCCM (Castilla-La Mancha, Spain).

\section{References}

[1] Fernández-Vicente, A., Carsí, M., Peñalba, F., Carreño, F. \& Ruano, O.A., Deformation behavior during hot torsion of and ultrahigh carbon steel containing 1.3 wt.\% C. Zeitschrift für Metallkunde, 94(8), pp. 922929, 2003. 
[2] Pantleon, W., Francke, D. \& Klimanek, P., Modelling adiabatic heating during high-speed deformation. Computational Materials Science, 7, pp. 75-81, 1996.

[3] Armstrong, R.W., Coffey, C.S. \& Elban, W.L., Adiabatic heating at a dislocation pile-up avalanche. Acta Metallurgica, 30, pp. 2111-2116, 1982.

[4] Prasad, Y.V.R.K., Gegel, H.L., Doraivelu, S.M., Malas, J.C., Morgan, J.T., Lark, K.A. \& Baker, D. R., Modelling of dynamic materials behavior in hot deformation: Forging of Ti-6242. Metallurgical Transactions A, 15A, pp. 1883-1892, 1984.

[5] Lindholm, U.S., Mechanical Properties at High Rates of Strain. Conference Series $n^{\circ} 21$, ed. J. Harding, Institute of Physics: London and Bristol, pp. 3-21, 1974.

[6] Bhattacharyya, A., Rittel, D. \& Ravichandran, G., Strain rate effect on the evolution of deformation texture for $\alpha$-Fe, Metallurgical and Materials Transactions A, 37(A), pp. 1137-1145, 2006

[7] Semiatin, S.L., Staker, M.R., \& Jonas, J.J., Plastic instability and flow localization in shear at high rates of deformation. Acta Metallurgica, 32 (9), pp. 1347-1354, 1984.

[8] Staker, M.R., The relation between adiabatic shear instability strain and material properties. Acta Metallurgica, 29, pp. 683-689, 1981

[9] Castellanos, J., Rieiro, I., Carsí, M, Muñoz, J., Ruano, O.A., Analysis of several methods for the data conversion and fitting of the Garofalo equation applied to an ultrahigh carbon steel. Journal of Achievements in Materials and Manufacturing Engineering, 18(1-2), pp. 447-454, 2006.

[10] Wei-Guo, G., Nemat-Nasser, S., Flow stress of Nitronic-50 stainless steel over a wide range of strain rates and temperatures. Mechanics of Materials, 38, pp. 1090-1103, 2006.

[11] Zhou, M., Clode, M.P., Thermal analysis of the torsion test under hotworking conditions. Computational Materials Science, 9, pp. 411-419, 1998.

[12] Holzer, A.J. \& Wright, P.K., Dynamic plasticity: a comparison between results from mechanical testing and machining. Materials Science and Engineering, 51, pp. 81- 92, 1981.

[13] Venugopal, P., Venugopal, S. \& Seetharaman, V., Some aspects of the dependence of the flow curve of commercially pure titanium on the forming temperature and the strain-rate. Journal of Materials Processing Technology, 21, pp. 201-217, 1990. 